\title{
Influence of serogroup B meningococcal vaccine antigens on growth and survival of the meningococcus in vitro and in ex vivo and in vivo models of infection
}

\author{
Kate L. Seib*, Francesca Oriente, Jeannette Adu-Bobie, Paolo Montanari, Francesca \\ Ferlicca, Marzia M. Giuliani, Rino Rappuoli, Mariagrazia Pizza and Isabel Delany
}

Novartis Vaccines, Via Fiorentina 1, 53100 Siena, Italy

*Corresponding author.

Mailing address: Molecular Genetics Unit, Novartis Vaccines, Via Fiorentina, 1, 53100 Siena, Italy. Phone: +39 0577245343. Fax:+39 0577243564. Email: Kate.Seib@novartis.com

\begin{abstract}
A novel vaccine against serogroup B meningococcal disease - containing a combination of protein antigens identified by reverse vaccinology: fHBP fused to GNA2091, GNA2132 fused to GNA1030, and NadA - is currently in Phase III clinical trials. In order to determine the role of these antigens in the growth, survival and fitness of the meningococcus, we generated a mutant lacking the expression of all five protein antigens $(5 \mathrm{KO})$, a mutant lacking the three main antigens (fHBP, GNA2132 and NadA; 3KO), as well as strains lacking the single antigens. Our results show that abrogation of expression of these antigens in Neisseria meningitidis results in reduced growth in vitro, increased sensitivity of the bacterium to stresses it may encounter in the host, as well as reduced fitness in ex vivo models of infection and in an in vivo infant rat competitive index assay. These results support a multivalent vaccine approach, which was undertaken to strengthen the protective activity of the vaccine antigens, increase the breadth of MenB strains targeted by the vaccine, and limit the potential for selection of vaccine escape mutants.
\end{abstract}

Keywords: meningococcal vaccine antigens; reverse vaccinology; fHBP; NadA; GNA2132; GNA2091; GNA1030.

Running title: Role of meningococcal vaccine antigens in survival and fitness 


\section{Introduction}

Neisseria meningitidis (the meningococcus) is an encapsulated Gram-negative diplococcus that colonizes the nasopharyngeal mucosa of approximately $5-10 \%$ of the population. However, the bacterium can also cross the epithelial layer into the bloodstream, leading to septicemia and/or meningitis [1]. Disease caused by $N$. meningitidis results in significant morbidity and mortality worldwide, with the majority of this disease being caused by five meningococcal serogroups (serogroups A, B, C, W-135 and Y). Conventional vaccinology approaches have led to the development of effective capsular-polysaccharide based vaccines against meningococcal serogroups A, C, W-135, Y [reviewed in 2]. However, there is still no broadly protective vaccine available against meningococcal serogroup $\mathrm{B}$ (MenB) disease, which is the main cause of meningococcal disease in many countries (approximately one-third of disease in the US [3] and more than half in Europe [4]). MenB vaccine candidates identified by conventional approaches have proven to be either (1) similar to self-antigens and as such poorly immunogenic (e.g. the capsule polysaccharide) or (2) hypervariable and/or poorly conserved between the diverse strains that cause endemic MenB disease (e.g. antigenically and phase variable outer membrane proteins and LPS) [5]. As a result of these limitations, the only currently available MenB vaccines are the "tailor-made" outer membrane vesicle (OMV) vaccines that have been developed and successfully used in New Zealand, Cuba and Norway to control epidemics that have primarily been caused by a single strain [6].

Recently, a multivalent recombinant vaccine against MenB, developed using genome-based "Reverse Vaccinology", has entered phase III clinical trials. The MenB vaccine contains 2 fusion proteins and one single polypeptide: factor $\mathrm{H}$-binding protein (fHBP; formerly GNA1870) fused to GNA2091; GNA2132 fused to GNA1030; and NadA (formerly GNA1994). These antigens were selected based on their ability to induce broad protection inferred by serum bactericidal activity (SBA) assays or by passive protection in infant rat or mouse models [7]. NadA, fHBP and GNA2132 are considered the main antigens, whereas GNA1030 and GNA2091 are regarded as accessory proteins, due to a less pronounced protective activity. The recombinant antigens are formulated with alum, with or without OMV from the New Zealand MeNZB ${ }^{\mathrm{TM}}$ vaccine strain that contains PorA 1.4 as the main immunogen [8]. When tested against a panel of 85 MenB strains representative of the global population of disease-causing strains, the combination of the recombinant antigens induced bactericidal antibodies in mice against $78 \%$ and $90 \%$ of strains, when administered with the adjuvants aluminum hydroxide and MF59 (an oil-in-water emulsion), respectively [7]. Phase II clinical results in infants indicated that this vaccine was well tolerated and induced a protective immune response against three diverse MenB strains in $89-96 \%$ of subjects following three vaccinations, and $93-100 \%$ after four vaccinations [9]. 
fHBP is a Neisseria-specific surface lipoprotein that binds human factor $\mathrm{H}(\mathrm{fH})$, a key inhibitor of the complement alternative pathway (AP). Binding of human $\mathrm{fH}$ to $\mathrm{fHBP}$ on the meningococcal surface allows the pathogen to evade complement-mediated killing by the innate immune system $[10,11]$. As a result, fHBP is important for survival of bacteria in human serum and blood, and is involved in protection against the antimicrobial peptide LL-37 via an unknown mechanism [10, 12, 13]. fHBP is expressed by all N. meningitidis strains studied to date [14] and can be classified into three variants (or two subfamilies) that are not cross protective [14, 15]. fHBP variant 1 , present in the MenB vaccine, is present in approximately $50-60 \%$ of disease-producing group B isolates $[16,17]$.

$\mathrm{NadA}$ is a surface-exposed trimeric autotransporter adhesin that is involved in adhesion and invasion of $N$. meningitidis into human epithelial cells $[18,19]$. Additional studies have shown that $\mathrm{NadA}$ is also involved in tissue and blood invasion as well as interacting with and stimulating immune cells during infection [20-22]. This antigen clusters into 3 main variants in virulent strains [16], and induces bactericidal antibodies that are not influenced by variant diversity. The nadA gene is present in approximately $50 \%$ of meningococcal isolates and is almost always present in three of the main hypervirulent lineages assigned by multilocus sequence type (MLST) [18, 23].

GNA2132 has recently been described as a heparin binding protein of N. meningitidis [24]. This antigen binds heparin at an arginine rich region and this interaction may increase bacterial serum resistance due to the potential interactions of heparin with components of the complement pathway, such as fH [24]. GNA2132 is cleaved by 2 different proteases, the Neisserial autotransporter lipoprotein NalP and human lactoferrin (Lf), and the functional significance of these cleavages is being studied. There are several variants of this protein [16] that are cross protective [7, 25].

To further increase the immunogenicity of the recombinant antigens, protein-protein fusions of fHBP and GNA2132 were generated with GNA2091 and GNA1030, respectively. GNA2091 was selected for its ability to induce protection in an mouse protection model, and GNA1030 for its ability to induce bactericidal antibodies against several strains. These proteins are well conserved in $N$. meningitidis strains, but are less well functionally characterized. The fusions with these proteins induce immune responses in SBA assays that are generally more potent than those induced by the individual antigens [7].

The inclusion of multiple antigens in the MenB vaccine should allow the breadth of MenB strains targeted by the vaccine to be increased, strengthen the protective activity of the vaccine, and reduce the risk of selecting for vaccine escape mutants (i.e. bacteria that have a mutation in a gene encoding an antigen that could allow them to avoid vaccine-induced bactericidal antibodies). To determine the contribution of each of the vaccine antigens in the growth and survival of $N$. 
meningitidis, and to evaluate the outcome of possible escape mutants lacking these vaccine antigens, MC58 derivative strains were generated that lacks the expression of all five antigens contained in the vaccine, a combination of the three main antigens, or each of the single antigens. These strains were analysed with respect to growth and survival in various media and stress conditions (including antimicrobial peptide, oxidative and envelope stress assays), in ex vivo human serum and whole blood models of meningococcal bacteremia and in vivo in the infant rat model of infection.

\section{Materials and Methods}

\subsection{Bacterial strains and culture conditions}

N. meningitidis strains used in this study are described in Table 1. N. meningitidis strains were routinely grown on GC (Difco) agar supplemented with Kellogg's supplement I, or on Mueller Hinton (MH) agar (Difco) at $37^{\circ} \mathrm{C} / 5 \% \mathrm{CO}_{2}$ overnight. For liquid cultures, overnight growth was used to inoculate GC broth supplemented with Kellogg's supplement I, $12.5 \mu \mathrm{M} \mathrm{Fe}\left(\mathrm{NO}_{3}\right)_{3}$, Catlin6 broth [26], MH broth or MH broth supplemented with $0.25 \%$ glucose. When required, tetracycline, erythromycin, chloramphenicol and kanamycin antibiotics were used at final concentrations of 2 $\mu \mathrm{g} / \mathrm{ml}, 5 \mu \mathrm{g} / \mathrm{ml}, 5 \mu \mathrm{g} / \mathrm{ml}$ and $100 \mu \mathrm{g} / \mathrm{ml}$, respectively. Escherichia coli strains used for cloning were cultured in Luria-Bertani (LB) broth or on LB agar (Difco). When required, erythromycin and tetracycline were used at a final concentration of $5 \mu \mathrm{g} / \mathrm{ml}$ and $10 \mu \mathrm{g} / \mathrm{ml}$, respectively and chloramphenicol and kanamycin at a final concentration of $20 \mu \mathrm{g} / \mathrm{ml}$.

Growth experiments were performed using a suspension of several colonies of bacteria taken from overnight plates, which were adjusted to an initial optical density at $600 \mathrm{~nm}\left(\mathrm{OD}_{600}\right)$ of 0.05 in $7 \mathrm{ml}$ of broth in $14 \mathrm{ml}$ tubes. The $\mathrm{OD}_{600}$ was then followed over time directly from the culture tubes. When shown, colony forming units (CFU) were also determined. Samples were taken at various times, from cultures grown in parallel, diluted and plated. Experiments were performed in triplicate and on several occasions.

\subsection{Recombinant DNA techniques}

Recombinant DNA techniques were routinely performed as described by Sambrook et al. [27]. Plasmid DNA preparations and purification of DNA fragments from PCR samples and agarose were performed using QIAGEN kits according to the manufacturer's instructions. PCR amplification was carried out in a $50 \mu \mathrm{l}$ reaction consisting of $1 \mathrm{x}$ reaction buffer, $1.5 \mathrm{mM} \mathrm{MgCl}{ }_{2}$, $0.2 \mathrm{mM}$ dNTPs, 1 unit of Platinum Taq polymerase (Invitrogen), and $10 \mathrm{pmol}$ of each forward and reverse primer, an appropriate amount of template DNA (chromosomal or plasmid DNA, or DNA from a single colony resuspended and boiled in $100 \mu \mathrm{l}$ water) and sterilized deionised water. All 
restriction endonucleases and T4 DNA ligase were obtained from New England Biolabs (NEB; Beverly, MA).

\subsection{Construction of knockout and complemented N. meningitidis strains}

In order to generate $N$. meningitidis mutant strains lacking the antigens contained in the MenB vaccine, five knockout plasmids were constructed to enable the deletion of all or part of the respective antigen-coding genes and replacement by allelic exchange with an antibiotic resistance cassette. Using standard cloning procedures, upstream and downstream flanking regions of the nmb2132, nmb1870, nmb1030, and $n m b 2091$ genes, (encoding the GNA2132, fHBP, GNA1030 and GNA2091 antigens respectively) were amplified by PCR from the MC58 genome and cloned on either side of an erythromycin resistance (Erm) cassette into the pBluescript (pBS; Pharmacia) cloning vector, generating the knockout plasmids pBSUD287::Erm, pBSUD741::Erm, pBSUD953::Erm, and pBSUD936::Erm, respectively. The upstream regions of the $n m b 2132$, nmb1870, nmb1030, and nmb2091 genes were amplified using primer pairs U287-F1/U287-R1, U741-F1/U741-R1, U953-F1/U953-R1, and U936-F1/U936-R1 (Table 2), respectively, and the downstream regions using U287-F1/U287-R1, U741-F1/U741-R1, U953-F1/U953-R1, and U936F1/U936-R1 (Table 2), respectively. For the generation of the NadA knockout construct, the nmb1994 gene was amplified by PCR from the MC58 genome using the primer pair 961-F3/961-R3 and cloned into the pBluescript cloning vector, and subsequently an internal HincII fragment at the 5' end of the coding region was substituted with the Erm cassette, generating pBS961::Erm. For generation of the single knockout mutants, the knockout plasmids were linearised and transformed into the MC58 wild type (WT) strain. Erythromycin resistant colonies were selected and checked by PCR for correct insertion due to a double homologous recombination event and colonies with correct PCR profile were further analysed by Western Blot for the lack of expression of the respective antigen. MC58 transformed with plasmids pBSUD287::Erm, pBSUD741::Erm, pBSUD953::Erm, pBSUD936::Erm and pBS961::Erm gave rise to strains $\triangle$ GNA2132, $\Delta$ fHBP, $\triangle$ GNA1030, $\Delta$ GNA2091 and $\triangle n a d A$ respectively. The $\triangle f H B P$ (previously called $\Delta g n a 1870$ ) mutant [14], and the $\triangle n a d A[19]$ have previously been described.

For generation of a single MC58 derivative strain lacking the expression of all five antigens, a stepwise deletion by allelic replacement strategy was used (Fig. 1A). First, an erythromycin sensitive $\Delta$ GNA2132 $\mathrm{erm}^{-}$single null mutant was generated by transformation of the $\Delta$ GNA2132 strain with the pBSUD287 plasmid containing contiguous upstream and downstream flanking regions of the $n m b 2132$ gene (without the Erm cassette) cloned into the pBS vector. Transformants were replica plated on GC agar with and without erythromycin selection, and erythromycin sensitive colonies were selected and checked by PCR for the correct double homologous 
recombination event leading to loss of the erythromycin cassette. The $\Delta$ GNA2132 $\mathrm{erm}^{-}$strain was $^{-}$ then transformed with the pBSUD741::Erm plasmid to generate the knockout of the $n m b 1870$ gene, and erythromycin resistant transformants were selected and checked by PCR and Western blot for the replacement of the $n m b 1870$ gene with the erythromycin resistant cassette giving rise to the double knockout strain $\triangle$ GNA2132 $\mathrm{erm}^{-} \triangle f H B P$ Erm. The stepwise knockout of the other three antigens was completed by substituting the erythromycin cassette in the pBS961::Erm, pBSUD936::Erm and pBSUD953::Erm knockout plasmid constructs with a chloramphenicol (Cm), a kanamycin (Kan) and a tetracycline (Tet) antibiotic resistance cassette, respectively, generating pBS961:Cm, pBSUD936::Kan and pBSUD953::Tet plasmids, respectively. This permitted the stepwise knockout of the three remaining antigen-expressing genes, via three consecutive transformations selecting for the appropriate antibiotic resistant colonies. Transforming the $\triangle \mathrm{GNA}^{2} 132 \mathrm{erm}^{-} \triangle f H B P$ Erm strain with $\mathrm{pBS} 961:: \mathrm{Cm}$ and selection of chloramphenicol resistant colonies lead to the generation of the MC58-3KO strain. Then transformation of the MC58-3KO strain with pBSUD936::Kan and selection of kanamycin resistant colonies lead to the isolation of the 4KO- $\triangle$ GNA2091Kan strain that was finally transformed with pBSUD953::Tet and selection on tetracycline lead to the isolation of the MC58-5KO strain. Each strain was checked by PCR for the correct double recombination at the allelic replacement locus and for the lack of expression of the appropriate antigens by Western Blot.

Complementation of the single null mutants was achieved by insertion of the deleted gene under the control of the $\mathrm{P}_{\text {tac }}$ promoter, along with the chloramphenicol resistance gene, into a noncoding chromosomal location between the two converging open reading frames (ORFs) NMB1428 and NMB1429, as previously described [13]. To complement the $\Delta$ GNA2091 strain, GNA2091 and $64 \mathrm{bp}$ of the non coding upstream region was amplified from MC58 using the primer pair 936compFOR3 and 936compREV3 (Table 2) and cloned as a NdeI-NsiI fragment into the pComPRBS plasmid. The addition of a short upstream nucleotide sequence of GNA2091 in the complemented strain enabled better restoration of expression. For complementation by allelic replacement, the null mutant was transformed with the respective complementation plasmid. Transformants were selected on chloramphenicol and checked by PCR, and complementation of the mutant strain was verified by Western blot analysis.

\subsection{Western blot analysis}

Expression of proteins in whole cell samples was performed essentially as described previously [14]. Briefly, strains were grown at $37^{\circ} \mathrm{C} / 5 \% \mathrm{CO}_{2}$ on agar plates overnight, or for time course experiments in broth from a starter inoculum of $\mathrm{OD}_{600} 0.05$ to stationary phase. Bacterial cells were resuspended in SDS-PAGE loading buffer in an appropriate calculated volume so as to 
normalize cell density to a final $\mathrm{OD}_{600}$ of 5 . Ponceau staining was performed to ensure equal loadings of the samples. Western blot analysis was performed according to standard procedures using polyclonal antibodies at a 1:1,000 dilution (raised in mice against the recombinant purified proteins, as described previously [14]), followed by a 1:5,000 dilution of HPR-labeled anti-mouse IgG (Sigma-Aldrich).

\subsection{In vitro antimicrobial peptide and environmental stress assays}

Overnight cultures were harvested into GC broth and diluted to approximately $10^{4} \mathrm{CFU} / \mathrm{ml}$. Each assay was started by the addition of $10 \mu \mathrm{l}$ of a compound to $90 \mu \mathrm{l}$ of the bacterial suspension. Compounds used are as follows: LL-37 (final concentration: 2-7 $\mu \mathrm{M}$; Innovagen, Sweden), detergent (0.01-0.1\% SDS, Tween, Triton X), reactive oxygen species (2-10 $\mathrm{mM} \mathrm{H}_{2} \mathrm{O}_{2}, 0.005$ $0.02 \%$ cumene hydroperoxide), or generators of reactive oxygen species $(2.5-10 \mathrm{mM}$ paraquat, xanthine $(1 \mathrm{mM}) / x a n t h i n e ~ o x i d a s e(200-400 \mathrm{mU})$ ). Samples were incubated at $37^{\circ} \mathrm{C} / 5 \% \mathrm{CO}_{2}$ with gentle agitation, and CFU were determined as described above. Sensitivity to osmotic stress was anlaysed with respect to the WT by growth in $\mathrm{GC}$ broth plus $\mathrm{NaCl}(0.3-0.5 \mathrm{M})$ or sucrose $(5-15 \%)$.

\subsection{Ex vivo human whole blood and human serum models of meningococcal bacteremia}

Cells were harvested into $\mathrm{MH}$ broth containing $0.25 \%$ glucose and $0.02 \mathrm{mM}$ Cytidine-5'monophospho-N-acetylneuraminic acid sodium salt (CMP-NANA) to an $\mathrm{OD}_{600}$ of 0.05 and grown to mid-log phase $\left(\mathrm{OD}_{600}\right.$ 0.5-0.6) then diluted in $\mathrm{MH}$ broth to $10^{3}-10^{6}$ colony forming units $(\mathrm{CFU}) / \mathrm{ml}$ depending on the bactericidal activity of the donor. The assay was started by the addition of $240 \mu 1$ whole human blood, human serum or heat inactivated human serum $\left(56{ }^{\circ} \mathrm{C} 30 \mathrm{~min}\right)$ to 10 $\mu \mathrm{l}$ of bacterial suspension. Cultures were incubated at $37^{\circ} \mathrm{C} / 5 \% \mathrm{CO}_{2}$ with gentle agitation, and at various time points an aliquot of the sample was removed and the number of viable CFU were determined by plating serial dilutions onto $\mathrm{MH}$ agar and incubating overnight at $37^{\circ} \mathrm{C} / 5 \% \mathrm{CO}_{2}$. Experiments were performed in triplicate on several occasions. The Student's t-test was used to determine the statistical significance of survival of each mutant strain with respect to the isogenic WT strain, with a p-value $<0.05$ considered to be significant. Whole venous blood, collected from healthy individuals (unimmunized against $N$. meningitidis) and anti-coagulated with heparin (10 $\mathrm{U} / \mathrm{ml}$ ), was used for whole blood experiments as described by Ison et al. [28]. For preparation of human serum, whole blood was coagulated at $25{ }^{\circ} \mathrm{C}$ for $30 \mathrm{~min}$, centrifuged at $1,000 \mathrm{xg}$ for $10 \mathrm{~min}$ at $4{ }^{\circ} \mathrm{C}$ and the supernatant retained. 


\subsection{In vivo infant rat model}

The infant rat model was used essentially as previously described [29], modified to include a competitive index (CI) analysis. Bacteria were grown to log phase $\left(\mathrm{OD}_{600}\right.$ of 0.25$)$ in $\mathrm{GC}$ medium, washed, and resuspended at the desired concentration in PBS. Five- to 6-day-old pups from litters of outbred Wistar rats (Charles River) were challenged intraperitoneally with the WT MC58 strain and the MC58-5KO strain together in a 1:1 ratio to establish mixed infections in the animals and determine the CI. Groups of 8 infant rats were used for each infectious dose of $1 \times 10^{5}, 1 \times 10^{6}$ and $1 \times 10^{7}$. A control group of 5 infant rats was injected with PBS. Eighteen hours after the bacterial challenge, blood samples were obtained by cheek puncture, and aliquots (100 $\mu 1$ of undiluted sera and $1: 10$ and $1: 1,000$ dilutions) were plated onto columbia agar $+5 \%$ horse blood and columbia agar $+5 \%$ horse blood plus erythromycin (to select for MC58-5KO bacteria) for viable cell counting. The numbers of $\mathrm{CFU} / \mathrm{ml}$ of blood were determined after overnight incubation of the plates at $37^{\circ} \mathrm{C}$ in an atmosphere containing $5 \% \mathrm{CO}_{2}$. Enumeration of WT bacteria and mutant bacteria allowed for the determination of the CI ratio using the following formula: $\mathrm{CI}=(\mathrm{WT}$ output/mutant output)/(WT input/mutant input). Statistical analysis was performed using the Wilcoxon Signed-Ranks Test.

\section{Results}

\subsection{Generation of meningococcal strains lacking expression of vaccine antigens}

N. meningitidis MC58 derivative strains were generated that lack the expression of all five of the antigens of the MenB vaccine (MC58-5KO), the three main antigens (MC58-3KO; lacking GNA2132, fHBP and NadA), as well as strains lacking the single antigens ( $\triangle \mathrm{fHBP}, \Delta \mathrm{NadA}$, $\Delta$ GNA2132, $\Delta$ GNA1030, $\Delta$ GNA2091). A strategy of stepwise deletion by allelic replacement was used to construct the MC58-3KO and MC58-5KO as described in the Materials and Methods. To allow the generation of the $5 \mathrm{KO}$ strain with the four antibiotic resistance cassettes available we started from a $\triangle$ GNA2132Erm MC58 strain and generated the $\triangle$ GNA2132erm ${ }^{-}$mutant lacking the Erm cassette. The $n m b 1870$ gene was then replaced with an Erm cassette, followed by replacement of $n a d A$ with a $\mathrm{Cm}$ cassette, replacement of the $n m b 2091$ gene with a Kan cassette, and finally the nmb1030 gene was replaced with a Tet cassette. Very few colonies were recovered exhibiting tetracycline resistance from this transformation. The deletion of each gene in the MC58-5KO strain was confirmed by PCR of each of the five loci (Fig. 1B) and Western blot analysis shows the absence of each protein in the intermediate and final knockout strains (Fig. 1C). The successful generation of a $5 \mathrm{KO}$ strain demonstrates that none of the genes are essential for in vitro growth of MC58 and moreover that the abrogation of the simultaneous expression of all of these proteins in the same strain gives rise to a viable mutant. 
Total protein samples taken during a time-course culture of the MC58 WT (Fig. 2A) indicates that each of the five proteins present in the MenB vaccine are expressed throughout the course of growth (Fig. 2B). More specifically, NadA is expressed at a lower level during log phase and increases during stationary phase [as previously seen by 18, 30]; GNA2132 is expressed in higher levels during log-phase than in stationary phase; while fHBP, GNA2091 and GNA1030 are expressed at constant levels throughout the different growth phases with a slight accumulation in stationary phase that is more pronounced for fHBP (Fig. 2B). As shown in Figure 1C, the deletion of the genes in the single knockouts or $3 \mathrm{KO}$ strain does not influence the level of expression of the other antigens.

\subsection{Characterization of growth in different media: GNA2091 is required for optimal growth}

In order to investigate the influence of the vaccine antigens in meningococcal growth and survival, we analysed growth of the MC58 WT and knockout strains in a series of different media. All single and multiple knockout strains generated were viable when grown in culture media including GC, Catlin6 and Mueller-Hinton (MH) broth, as well when plated on GC or MH agar. All knockout strains displayed colony morphology similar to the WT strain when grown on GC agar, and growth in GC broth is equivalent in all strains ( 46 min doubling time for $\mathrm{WT}, 3 \mathrm{KO}$ and $5 \mathrm{KO}$; Fig. 3A). Growth in chemically defined Catlin6 broth is also essentially equivalent in all strains, with slightly slower growth seen for the $5 \mathrm{KO}(\sim 69 \mathrm{~min}$ doubling time for WT, $75 \mathrm{~min}$ for $5 \mathrm{KO}$; Fig. 3B). However, the $5 \mathrm{KO}$ strain has smaller colony morphology when grown on $\mathrm{MH}$ agar and decreased growth in $\mathrm{MH}$ broth, with respect to the WT and the 3KO strains (doubling times: WT, $88 \mathrm{~min}$; 3KO, $89 \mathrm{~min}$; $5 \mathrm{KO} 97 \mathrm{~min}$; Fig. 3C). In particular, a similar lag phase was seen, but the growth rate of the $5 \mathrm{KO}$ slows during the exponential phase and it does not reach a cell density equivalent to that of the WT, as measured by $\mathrm{OD}_{600}$ and $\mathrm{CFU}$ count (Fig. 3C-D).

Further investigation revealed the growth phenotype of the $5 \mathrm{KO}$ strain to be a result of the lack of GNA2091, with the $\triangle$ GNA2091 strain displaying decreased growth in $\mathrm{MH}$ that was restored to near WT levels in the complemented strain, MC58 2091 C, in which the gene encoding GNA2091 is inserted in trans (doubling times: WT, $80 \mathrm{~min}$; $\Delta$ GNA2091, $104 \mathrm{~min}$; $\Delta$ GNA2091_C, $83 \mathrm{~min}$; Fig. 3E). The growth phenotype of $\triangle$ GNA2091 could be partially rescued by supplementing $\mathrm{MH}$ with $0.25 \%$ glucose (doubling times: WT, $71 \mathrm{~min} ; \Delta 2091,74 \mathrm{~min}$; Fig. 3F). Similarly, the growth phenotype of $\triangle$ GNA2091 could be partially rescued by supplementing MH with maltose, a disaccharide compose of two glucose monomers (doubling times: WT, $67 \mathrm{~min}$; $\Delta$ GNA2091, 67 min; data not shown). Western blot analysis of the MC58 WT strain grown in MH or MH plus glucose (0.25-1\%) showed equivalent expression of GNA2091 under conditions of low and high glucose, indicating that this gene is not regulated by glucose levels (data not shown). Investigation 
of this phenotype in $\triangle$ GNA2091 knockouts of additional meningococcal strains, including 67/00, NZ98/253 and 2996, revealed that GNA2091 is also required for optimal growth in these strains (data not shown).

\section{3. in vitro survival; $f H B P$ and GNA2091 play a role in various stress responses}

In order to determine whether the vaccine proteins are important for survival under various conditions, we performed several in vitro stress assays. Survival of MC58, the $3 \mathrm{KO}$ and the $5 \mathrm{KO}$ was investigated in the presence of: (1) oxidative stress to mimic the oxidative burst of phagocytic cells; (2) the antimicrobial peptide LL-37; and (3) envelope stress using detergent (SDS and Tween) and osmotic stress $(\mathrm{NaCl}$ and sucrose).

In a control experiment, the $\mathrm{WT}$, the $3 \mathrm{KO}, 5 \mathrm{KO}$ and single mutant strains all behave in a similar manner when incubated for the duration of the assays in GC broth, the media used to grow and dilute the cells for these assays (2-3 fold increase in CFU over 2 hours; data not shown). This indicates that all differences observed are not due to growth differences during the assay. No mutant strains displayed increased sensitivity with respect to the WT strain during the in vitro oxidative stress assays (including paraquat, hydrogen peroxide and xanthine/xanthine oxidase assays; data not shown).

The $5 \mathrm{KO}$ and $3 \mathrm{KO}$ strains are sensitive to the antimicrobial peptide LL-37, which is a part of the innate immune system that is constitutively produced by leukocytes (Fig. 4A). As previously reported [13], the $\triangle f H B P$ strain is sensitive to LL-37 and the phenotype of the $3 \mathrm{KO}$ and $5 \mathrm{KO}$ strains can be attributed to the lack of fHBP in these strains (Fig. 4A), rather than to the absence of other proteins since all other single KOs had survival equivalent to that of the WT (data not shown).

The $5 \mathrm{KO}$ strain (and to a lesser extent $3 \mathrm{KO}$ ) showed increased sensitivity to the anionic detergent SDS with respect to the WT, suggesting this strain may have increased membrane permeability (Fig. 4B). Analysis of the $\triangle$ GNA2091 single knockout strain revealed that this strain has increased sensitivity to SDS, which could be overcome when the respective knockout strain was complemented by inserting the gene in trans (Fig. 4C). The $\triangle$ GNA2091 knockout also has increased sensitivity to the non-ionic detergent Tween (data not shown). The 5KO strain did not show increased sensitivity to osmotic stress with respect to the WT when grown in GC broth plus $\mathrm{NaCl}$ or sucrose (data not shown).

\subsection{Survival of N. meningitidis in ex vivo human whole blood and human serum models of meningococcal bacteremia}

N. meningitidis MC58 WT, the 3KO and 5KO strains, as well as all single knockout strains were analysed in ex vivo human whole blood and human serum models of meningococcal 
bacteremia in order to determine the influence of the antigens on meningococcal survival and to ensure that the mutant strains do not have increased fitness in these models of infection. The human whole blood assay was used to assess cellular and humoral mechanisms of killing (i.e., complement activity, antibody-mediated serum bactericidal activity (SBA) and opsonophagocytosis (OP), as well as neutrophil, macrophage and antimicrobial peptide killing), while the serum assay was used to assess bacterial killing mediated by the humoral immune response. The $3 \mathrm{KO}, 5 \mathrm{KO}$ and the $\Delta$ fHBP knockout strains are highly sensitive to killing by both human whole blood (Fig. 5A) and serum (Fig. 6A) with 10-100 fold less survival for the mutant strains with respect to WT over two hours. The sensitivity of the $\triangle \mathrm{fHBP}$ knockout strain is consistent with its reported ability to bind factor $\mathrm{H}(\mathrm{fH})$, a key negative regulator of the alternative complement pathway $[13,31]$. On the other hand, the remaining four single knockout strains ( $\operatorname{nadA}, \Delta \mathrm{GNA} 2132, \Delta$ GNA2091 and $\Delta$ GNA1030) all display equivalent or slightly reduced (typically 2-5 fold lower) levels of survival with respect to the WT in both human whole blood (Fig. 5B) and serum (Fig. 6B), with differences seen between different donors. For example, $\Delta$ GNA2091 had decreased survival in the blood of donors 1 and 4 , and the serum of donors 3 and 4. $\Delta$ nadA had decreased survival in the blood of donor 4, and the serum of donors 1 and 3. $\triangle$ GNA2132 and $\triangle$ GNA1030 both had decreased survival in the serum of donor 2. The WT, all single mutants, as well as the $3 \mathrm{KO}$ and $5 \mathrm{KO}$ strains all behave in a similar manner when incubated for the duration of the assay in heat-inactivated serum (no growth or killing seen; data not shown).

\subsection{In vivo infant rat competition assay}

In order to examine the fitness of the MC58-5KO strain in vivo, a competitive index (CI) assay was performed in infant rats to determine if the WT bacteria could out-compete the $5 \mathrm{KO}$ bacteria. WT MC58 and 5KO bacteria were injected i.p. at a ratio of 1:1, at final concentrations of $1 \times 10^{5}, 1 \times 10^{6}$ or $1 \times 10^{7} \mathrm{CFU}$. Bacterial CFU in the blood were determined by replicate plating of serial dilutions on columbia agar and columbia agar plus erythromycin (to select for the $5 \mathrm{KO}$ strain). Figure 7 shows the CI of each infant rat for each infectious dose used. A CI of greater than 1 was seen for all rats in each group (median/average CI of 10.5/18.8, 4.2/6.8 and 3.6/10 for 1x10 $1 \times 10^{6}$ and $1 \times 10^{7}$ respectively) indicating that more WT bacteria survived in the infant rat model of infection than did mutant $5 \mathrm{KO}$ bacteria. This suggests that the lack of expression of the vaccine antigens in the $5 \mathrm{KO}$ strain significantly affects its ability to survive in vivo.

\section{Discussion}

In this study we evaluated the influence of the investigational Novartis multivalent MenB vaccine antigens in meningococcal growth and survival. The successful generation of a MC58-5KO 
strain, in which each of the antigen encoding genes had been genetically removed, demonstrates that none of the genes are essential for growth of the MC58 strain in vitro nor do they give synergistically negative effects on viability as the abrogation of expression of all of these proteins in the same strain gives rise to a viable mutant. The ability of meningococci to colonize the nasopharyngeal mucosa as well as to survive and multiply within human blood are key factors in the development of fulminant meningococcal disease. Hence we endeavored to evaluate the role of the vaccine antigens in growth and survival in various assays that mimic environmental stresses that the meningococcus may encounter during colonization and infection.

N. meningitidis MC58-5KO and $\Delta$ GNA20291 mutant strains have decreased growth capabilities in vitro with respect to the WT strain in $\mathrm{MH}$ broth and agar, which can be rescued by the addition of glucose and maltose to the media. The decreased growth of these strains may be relevant in the context of in vivo colonization of the nasopharynx, since saliva and nasal and airway secretions of healthy adults contain very low levels of glucose (10-100 fold less that blood) [32]. Furthermore, the MC58-5KO and $\triangle$ GNA2091 mutant strains are susceptible to detergent stress. The detergents SDS and Tween were used to mimic membrane stress, which the meningococcus may encounter in the host during colonization or invasive disease. For example, innate host defense of the nasal/nasopharyngeal mucosa include many compounds that disrupt the integrity of bacterial membranes [33], including surfactant protein $\mathrm{D}$, which is known to play a role in inhibiting nasopharyngeal colonization of the pneumococcus [34].

The MC58-5KO and -3KO strains showed a dramatically increased susceptibility to killing by factors of the human immune response, as displayed in the in vitro LL-37 antimicrobial peptide killing assays, and the ex vivo human serum and whole blood models of meningococcal bacteremia. This sensitivity can primarily be attributed to the lack of fHBP in these strains, as the $\Delta \mathrm{fHBP}$ strain has a similar phenotype. N. meningitidis has developed many mechanisms to evade the human immune response [35] and fHBP is emerging as a key player in mediating resistance to host defenses, due to its ability to bind $\mathrm{fH}$, an inhibitory regulator of the alternative complement pathway. The importance of the complement pathway in preventing development of meningococcal disease is evidenced by the increased incidence and recurrence of infection and disease in people with immune disorders related to $\mathrm{fH}$ [36-39]. fHBP elicits a strong direct bactericidal response through SBA and OP $[12,14]$, and anti-fHBP antibodies are able to block binding of fH to the meningococcal surface, increasing susceptibility to killing $[10,40]$. fHBP is expressed by almost all N. meningitidis strains studied to date, however the level of expression varies between strains (high, intermediate, or low expressors) $[14,41]$ MC58 is a ST-32 strain that has been characterized as a high expressor of fHBP and is a strain that is dependent on fHBP for survival in human blood and serum $[10,12,13]$ and such high levels of fHBP may mask subtle roles played by other proteins. It 
is important to note that some of the other single knockout strains also displayed a small increase in sensitivity in the blood and serum assay, suggesting that they may play a role in survival in vivo in the human host. This may be particularly relevant in strains that express less FHBP, and that may be more dependent on other antigens for survival. Furthermore, in the in vivo infant rat model used to investigate the competitive index of the WT and the $5 \mathrm{KO}$ strains, the WT was seen to out-compete the mutant strain in all infected animals when an infectious dose of $1 \times 10^{5}$ or $1 \times 10^{6} \mathrm{CFU}$ was used. Since fHBP binds specifically to human, but not rat factor $\mathrm{H}$ [42], this decreased survival of the $5 \mathrm{KO}$ in vivo cannot be attributed to the lack of fHBP. It however strongly suggests that the expression of the one or more of the vaccine antigens plays an important role in the growth, fitness and/and survival of the meningococcus in vivo in this model of infection. Further experiments will be needed to elucidate the mechanism by which these antigens contribute to survival in this model of infection.

We have shown that the proteins present in the MenB vaccine are expressed throughout the in vitro growth cycle. It has also recently been shown that the level of antigen expression, and sensitivity to SBA, does not change after up to 5 rounds of selection in the presence of bactericidal antibodies generated in mice against the MenB antigens [43]. Since these proteins are important for growth and survival, they are likely to be expressed in vivo during colonization and/or infection, and indeed antibodies against NadA, fHBP and GNA2132 are present children convalescing after meningococcal disease, and antibody levels tend to increase with age [24, 44, 45]. Furthermore, the level of expression of these antigens may be higher in vivo than in vitro. For example, transcription of fHBP is increased during oxygen limitation in an FNR-dependent manner that may be relevant for certain microenvironments in the host, such as the submucosa or intracellular niches [46]. NadA is repressed by NadR [30,47], but is derepressed in the presence of 4-hydroxyphenylacetic acid, a metabolite of aromatic amino acid catabolism that is secreted in saliva [30]. As such, NadA may be induced during colonization of the nasopharynx enabling it to mediate adhesion and invasion of the mucosal epithelium. Furthermore, it has been proposed that anti-NadA antibodies may be able to decrease adherence to and invasion of host epithelial cells, since a NadA mutant strain has decreased association and invasion of epithelial cells [19]. Likewise, there is a possibility that antiGNA2132 antibodies may interfere with its role in heparin binding, rendering it more susceptible to killing. It could also be speculated that anti-GNA2091 antibodies may play a role in decreasing growth of the bacteria in low glucose environments in the host. Unfortunately, no appropriate model is available to evaluate meningococcal colonization.

In summary, the abrogation of expression of the MenB vaccine antigens results in reduced growth in vitro, and significantly affects sensitivity of the meningococcus to stresses it may encounter in the host, as well as reducing its fitness in ex vivo models of infection. These findings 
support the multivalent vaccine approach that has been used, and supports the idea that the potential for selection of vaccine escape mutants should be limited by using this combination of antigens.

\section{Acknowledgements}

KLS is the recipient of an Australian NHMRC CJ Martin fellowship. FO is the recipient of a Novartis fellowship from the PhD program in Evolutionary Biology of the University of Siena. We thank Davide Serruto, Beatrice Aricò, Vincenzo Scarlato, and Richard Moxon for useful discussion. We thank the animal care facility at Novartis Vaccines for technical assistance.

\section{References}

[1] Rosenstein NE, Perkins BA, Stephens DS, Popovic T, Hughes JM. Meningococcal disease. N Engl J Med 2001 May 3;344(18):1378-88.

[2] Stephens DS. Conquering the meningococcus. FEMS Microbiol Rev 2007 Jan;31(1):3-14.

[3] Rosenstein NE, Perkins BA, Stephens DS, Lefkowitz L, Cartter ML, Danila R, et al. The changing epidemiology of meningococcal disease in the United States, 1992-1996. J Infect Dis 1999 Dec;180(6):1894-901.

[4] Noah N, Henderson B. Surveillance of Bacterial Meningitis in Europe 1997/1998: Communicable Disease Surveillance Centre, London; 2002.

[5] Seib KL, Rappuoli R. Difficulties in developing neisserial vaccines. In: Genco CA, Wetzler LM, editors. Neisseria: Molecular Mechanisms of Pathogenesis. Norwich, UK: Horizon Scientific Press, 2008: In press.

[6] Holst J, Martin D, Arnold R, Huergo CC, Oster P, O'Hallahan J, et al. Properties and clinical performance of vaccines containing outer membrane vesicles from Neisseria meningitidis. Vaccine 2009;27(Supplement 2):B3-B12.

[7] Giuliani MM, Adu-Bobie J, Comanducci M, Arico B, Savino S, Santini L, et al. A universal vaccine for serogroup B meningococcus. Proceedings of the National Academy of Sciences of the United States of America 2006 Jul 18;103(29):10834-9.

[8] Martin DR, Ruijne N, McCallum L, O'Hallahan J, Oster P. The VR2 epitope on the PorA $\mathrm{P} 1.7-2,4$ protein is the major target for the immune response elicited by the strain-specific group B meningococcal vaccine MeNZB. Clin Vaccine Immunol 2006 Apr;13(4):486-91. 
[9] Rappuoli R. The application of reverse vaccinology, Novartis MenB vaccine developed by design. 16th International Pathogenic Neisseria Conference Rotterdam, The Netherlands: www.IPNC2008.org; Abstr. , p. 81, 2008.

[10] Madico G, Welsch JA, Lewis LA, McNaughton A, Perlman DH, Costello CE, et al. The meningococcal vaccine candidate GNA1870 binds the complement regulatory protein factor $\mathrm{H}$ and enhances serum resistance. J Immunol 2006 Jul 1;177(1):501-10.

[11] Granoff DM, Welsch JA, Ram S. Binding of complement factor H (fH) to Neisseria meningitidis is specific for human $\mathrm{fH}$ and inhibits complement activation by rat and rabbit sera. Infection and immunity 2009 Feb;77(2):764-9.

[12] Welsch JA, Ram S, Koeberling O, Granoff DM. Complement-dependent synergistic bactericidal activity of antibodies against factor H-binding protein, a sparsely distributed meningococcal vaccine antigen. J Infect Dis 2008 Apr 1;197(7):1053-61.

[13] Seib KL, Serruto D, Oriente F, Delany I, Adu-Bobie J, Veggi D, et al. Factor H-binding protein is important for meningococcal survival in human whole blood and serum and in the presence of the antimicrobial peptide LL-37. Infection and immunity 2009 Jan;77(1):292-9.

[14] Masignani V, Comanducci M, Giuliani MM, Bambini S, Adu-Bobie J, Arico B, et al. Vaccination against Neisseria meningitidis using three variants of the lipoprotein GNA1870. J Exp Med 2003 Mar 17;197(6):789-99.

[15] Giuliani MM, Santini L, Brunelli B, Biolchi A, Arico B, Di Marcello F, et al. The region comprising amino acids 100 to 255 of Neisseria meningitidis lipoprotein GNA 1870 elicits bactericidal antibodies. Infection and immunity 2005 Feb;73(2):1151-60.

[16] Bambini S, Muzzi A, Olcen P, Rappuoli R, Pizza M, Comanducci M. Distribution and genetic variability of three vaccine components in a panel of strains representative of the diversity of serogroup B meningococcus. Vaccine 2009;27(21):2794-803.

[17] Welsch JA, Rossi R, Comanducci M, Granoff DM. Protective activity of monoclonal antibodies to genome-derived neisserial antigen 1870, a Neisseria meningitidis candidate vaccine. J Immunol 2004 May 1;172(9):5606-15.

[18] Comanducci M, Bambini S, Brunelli B, Adu-Bobie J, Arico B, Capecchi B, et al. NadA, a novel vaccine candidate of Neisseria meningitidis. J Exp Med 2002 Jun 3;195(11):1445-54.

[19] Capecchi B, Adu-Bobie J, Di Marcello F, Ciucchi L, Masignani V, Taddei A, et al. Neisseria meningitidis NadA is a new invasin which promotes bacterial adhesion to and penetration into human epithelial cells. Molecular microbiology 2005 Feb;55(3):687-98. 
[20] Franzoso S, Mazzon C, Sztukowska M, Cecchini P, Kasic T, Capecchi B, et al. Human monocytes/macrophages are a target of Neisseria meningitidis Adhesin A (NadA). J Leukoc Biol 2008 May;83(5):1100-10.

[21] Tavano R, Franzoso S, Cecchini P, Cartocci E, Oriente F, Arico B, et al. The membrane expression of Neisseria meningitidis adhesin A (NadA) increases the proimmune effects of MenB OMVs on human macrophages, compared with NadA- OMVs, without further stimulating their proinflammatory activity on circulating monocytes. J Leukoc Biol 2009 Jul;86(1):143-53.

[22] Mazzon C, Baldani-Guerra B, Cecchini P, Kasic T, Viola A, de Bernard M, et al. IFN\{gamma\} and R-848 Dependent Activation of Human Monocyte-Derived Dendritic Cells by Neisseria meningitidis Adhesin A. J Immunol 2007 Sep 15;179(6):3904-16.

[23] Comanducci M, Bambini S, Caugant DA, Mora M, Brunelli B, Capecchi B, et al. NadA diversity and carriage in Neisseria meningitidis. Infection and immunity 2004 Jul;72(7):421723.

[24] Serruto D, Spadafina T, Ciucchi L, Lewis LA, Ram S, Tontini M, et al. Neisseria meningitidis GNA2132 is a heparin binding protein cleaved by NalP and human lactoferrin serine proteases. 2009;manuscript in preparation.

[25] Welsch JA, Moe GR, Rossi R, Adu-Bobie J, Rappuoli R, Granoff DM. Antibody to genomederived neisserial antigen 2132, a Neisseria meningitidis candidate vaccine, confers protection against bacteremia in the absence of complement-mediated bactericidal activity. J Infect Dis 2003 Dec 1;188(11):1730-40.

[26] Fu J, Bailey FJ, King JJ, Parker CB, Robinett RS, Kolodin DG, et al. Recent advances in the large scale fermentation of Neisseria meningitidis group B for the production of an outer membrane protein complex. Biotechnology (N Y) 1995 Feb;13(2):170-4.

[27] Sambrook J, Fritsch EF, Maniatis T. Molecular cloning: A laboratory manual. 2nd ed: Cold Spring Harbor, NY: Cold Spring Harbor Laboratory., 1989.

[28] Ison CA, Anwar N, Cole MJ, Galassini R, Heyderman RS, Klein NJ, et al. Assessment of immune response to meningococcal disease: comparison of a whole-blood assay and the serum bactericidal assay. Microb Pathog 1999 Oct;27(4):207-14.

[29] Granoff DM, Moe GR, Giuliani MM, Adu-Bobie J, Santini L, Brunelli B, et al. A novel mimetic antigen eliciting protective antibody to Neisseria meningitidis. J Immunol 2001 Dec 1;167(11):6487-96. 
[30] Metruccio MME, Pigozzi E, Roncarati D, Berlanda Scorza F, Norais N, Hill SA, et al. A novel phase variation mechanism in the meningococcus driven by a ligand-responsive repressor and differential spacing of distal promoter elements. PLoS Pathog 2009; In press.

[31] Mader R, Zu'bi A, Schonfeld S. Recurrent sterile arthritis following primary septic meningococcal arthritis. Clin Exp Rheumatol 1994 Sep-Oct;12(5):531-3.

[32] Philips B, Meguer J-X, Redman J, Baker E. Factors determining the appearance of glucose in upper and lower respiratory tract secretions. Intensive Care Medicine 2003;29(12):2204-10.

[33] Rogan MP, Geraghty P, Greene CM, O'Neill SJ, Taggart CC, McElvaney NG. Antimicrobial proteins and polypeptides in pulmonary innate defence. Respir Res 2006;7(1):29.

[34] Jounblat R, Clark H, Eggleton P, Hawgood S, Andrew PW, Kadioglu A. The role of surfactant protein $\mathrm{D}$ in the colonisation of the respiratory tract and onset of bacteraemia during pneumococcal pneumonia. Respir Res 2005;6:126.

[35] Meyer TF. Evasion mechanisms of pathogenic Neisseriae. Behring Inst Mitt 1991 Feb;88:194-9.

[36] Nielsen HE, Christensen KC, Koch C, Thomsen BS, Heegaard NH, Tranum-Jensen J. Hereditary, complete deficiency of complement factor $\mathrm{H}$ associated with recurrent meningococcal disease. Scand J Immunol 1989 Dec;30(6):711-8.

[37] Ram S, Vogel U. Role of complement in defence against meningococcal infection. In: Frosch M, Maiden MCJ, editors. Handbook of Meningococcal Disease Infection Biology, Vaccination, Clinical Management. Weinheim: Wiley-VCH Verlag GmbH \& Co., 2006: 27393.

[38] Mathew S, Overturf GD. Complement and properidin deficiencies in meningococcal disease. Pediatr Infect Dis J 2006 Mar;25(3):255-6.

[39] Haralambous E, Dolly SO, Hibberd ML, Litt DJ, Udalova IA, O'Dwyer C, et al. Factor H, a regulator of complement activity, is a major determinant of meningococcal disease susceptibility in UK Caucasian patients. Scand J Infect Dis 2006;38(9):764-71.

[40] Beernink PT, Welsch JA, Bar-Lev M, Koeberling O, Comanducci M, Granoff DM. Fine Antigenic Specificity and Cooperative Bactericidal Activity of Monoclonal Antibodies Directed at the Meningococcal Vaccine Candidate, Factor H-Binding Protein. Infection and immunity 2008 Jun 30;76(9):4232-40. 
[41] McNeil LK, Murphy E, Zhao XJ, Guttmann S, Harris S, Scott A, et al. Detection of LP2086 on the cell surface of Neisseria meningitidis and its accessibility in the presence of serogroup B capsular polysaccharide. Vaccine 2009 Feb 4.

[42] Schneider MC, Exley RM, Ram S, Sim RB, Tang CM. Interactions between Neisseria meningitidis and the complement system. Trends Microbiol 2007 May;15(5):233-40.

[43] Santos GF, Giuliani M, Santini L, Adu-Bobie J, Pizza M, Rappuoli R, et al. Investigation on the effect of immune selection on resistance to bactericidal antibodies to group $\mathrm{B}$ meningococci in vitro. Clin Vaccine Immunol 2009 Nov;16(11):1693-5.

[44] Litt DJ, Savino S, Beddek A, Comanducci M, Sandiford C, Stevens J, et al. Putative vaccine antigens from Neisseria meningitidis recognized by serum antibodies of young children convalescing after meningococcal disease. J Infect Dis 2004 Oct 15;190(8):1488-97.

[45] Jacobsson S, Molling P, Olcen P. Seroprevalence of antibodies against fHbp and NadA, two potential vaccine antigens for Neisseria meningitidis. Vaccine 2009 Aug 1.

[46] Oriente F, Scarlato V, Delany I. Expression of factor H binding protein of meningococcus responds to oxygen-limitation through a dedicated Fnr-regulated promoter. In preparation 2009.

[47] Schielke S, Huebner C, Spatz C, Nagele V, Ackermann N, Frosch M, et al. Expression of the meningococcal adhesin NadA is controlled by a transcriptional regulator of the MarR family. Molecular microbiology 2009 May;72(4):1054-67.

[48] Tettelin H, Saunders NJ, Heidelberg J, Jeffries AC, Nelson KE, Eisen JA, et al. Complete genome sequence of Neisseria meningitidis serogroup B strain MC58. Science 2000 Mar 10;287(5459):1809-15. 
Table 1. N. meningitidis strains used in this study

\begin{tabular}{|c|c|c|}
\hline Strain & Description & Reference \\
\hline MC58 & $\begin{array}{l}\text { Clinical isolate from the United Kingdom (1985) for which the } \\
\text { genome sequence is available. } \\
\text { Classification: B:15:P1.7, 16; ET5; ST-74. }\end{array}$ & {$[48]$} \\
\hline MC58-3KO & $\begin{array}{l}\text { Mutant derivative of MC58 in which the NMB2132 gene has } \\
\text { been substituted by Erm; Erm removed from the NMB2132 } \\
\text { gene; the NMB1870 gene has been substituted by Erm; and the } \\
\text { NMB1994 gene has been substituted by Cm. }\end{array}$ & $\begin{array}{l}\text { This study } \\
\text { see Fig. } 1 .\end{array}$ \\
\hline MC58-5KO & $\begin{array}{l}\text { Mutant derivative of the MC58 3KO strain in which the } \\
\text { NMB2091 gene has been substituted by Kan; and the NMB1030 } \\
\text { gene has been substituted by Tet. }\end{array}$ & $\begin{array}{l}\text { This study } \\
\text { see Fig. } 1 .\end{array}$ \\
\hline$\triangle$ GNA2132 & $\begin{array}{l}\text { GNA2132 mutant derivative of MC58 in which the NMB2132 } \\
\text { gene has been substituted by Erm. }\end{array}$ & {$[24]$} \\
\hline$\triangle f H B P$ & $\begin{array}{l}\text { fHBP mutant derivative of MC58 in which the NMB1870 gene } \\
\text { is replaced by Erm. }\end{array}$ & {$[14]$} \\
\hline$\Delta n a d A$ & $\begin{array}{l}\text { NadA mutant derivative of MC58 in which the NMB1994 gene } \\
\text { has been interrupted with Erm. }\end{array}$ & {$[19]$} \\
\hline$\triangle$ GNA2091 & $\begin{array}{l}\text { GNA2091 mutant derivative of MC58 in which the NMB2091 } \\
\text { gene has been interrupted with Erm. }\end{array}$ & This study \\
\hline$\Delta$ GNA1030 & $\begin{array}{l}\text { GNA1030 mutant derivative of MC58 in which the NMB1030 } \\
\text { gene has been interrupted with Erm. }\end{array}$ & This study \\
\hline$\Delta$ GNA2091_C & $\begin{array}{l}\text { Complemented GNA2091 strain; derivative of } \Delta \text { GNA2091 in } \\
\text { which the NMB2091 gene under the control of the heterologous } \\
\mathrm{P}_{\text {tac }} \text { promoter are inserted, with Cm into the noncoding region } \\
\text { between NMB1428 and NMB1429. }\end{array}$ & This study \\
\hline
\end{tabular}

3KO, 3 knockout strain; 5KO, 5 knockout strain; Erm, erythromycin resistance cassette; Cm, erythromycin resistance cassette; Kan, kanamycin resistance cassette; Tet, tetracycline resistance cassette. 
Table 2. Oligonucleotides used in this study

\begin{tabular}{|c|c|c|}
\hline Name & Sequence $^{\mathrm{a}}$ & Site $^{b}$ \\
\hline $\mathrm{U} 741-\mathrm{F} 1$ & gctctagaCCAGCCACGGCGCATAC & XbaI \\
\hline \begin{tabular}{|l|} 
U741-R1 \\
\end{tabular} & tcccceggg GACGGCATTTTGTTTACAGG & Smal \\
\hline D741-F1 & tcceccggg CGCCAAGCAATAACCATTG & Smal \\
\hline D741-R1 & cccgctcgagCAGCGTATCGAACCATGC & XhoI \\
\hline $961-\mathrm{F} 3$ & gctctagaGAAACACTTTCCATCCAAAG & XbaI \\
\hline $961-\mathrm{R} 3$ & ccgctcgagTTACCATCGTAATTGACG & XhoI \\
\hline U936-F1 & gctctagaGCCTGATGAATGACGGC & XbaI \\
\hline U936-R1 & tccccegggGGTTTCATCGGGTGCTTCC & Smal \\
\hline D936-F1 & tccccegggCTACGTCCAACGCTGACTC & Smal \\
\hline D936-R1 & cccgctcgagGCTTGTGTTCCGGCGTCG & XhoI \\
\hline U287-F1 & gctctagaGGCGTTTATGCCTTCTTTAC & XbaI \\
\hline U287-R1 & tccccccgggCATCATCTCCTTCATCGTATT & Smal \\
\hline D287-F1 & tcccccgggGGATTGATGTTGATGCCG & Smal \\
\hline D287-R1 & ccgctcgagGGGTATCTACTCGCAAAG & XhoI \\
\hline U953-F1 & gctctagaGAACTCACCGGCGACATC & XbaI \\
\hline U953-R1 & tcceccgggGCAGTACTGATGGCGG & Smal \\
\hline D953-F1 & tcccccgggCATCGACATCCAAATCGAG & Smal \\
\hline D953-R1 & cccgctcgagCGATTGGCTGGCATTGAC & XhoI \\
\hline 936compFOR3 & gggaattccatatgACCCTTTTCAGACGGCATGG & NdeI \\
\hline 936compREV3 & ccaatgcatTCAGCGTTGGACGTAGTTTTGG & NsiI \\
\hline
\end{tabular}

${ }^{\text {a }}$ Capital letters correspond to nucleotides of the MC58 genome sequence and small letters to either nucleotides of $E$. coli genome or plasmid sequence origin or nucleotides added for cloning reasons, underlined letters indicate sequences of restriction enzyme sites used for cloning PCR fragments.

${ }^{\mathrm{b}}$ Enzymes for which the restriction sites are present in the sequence of the primer, added for cloning reasons 

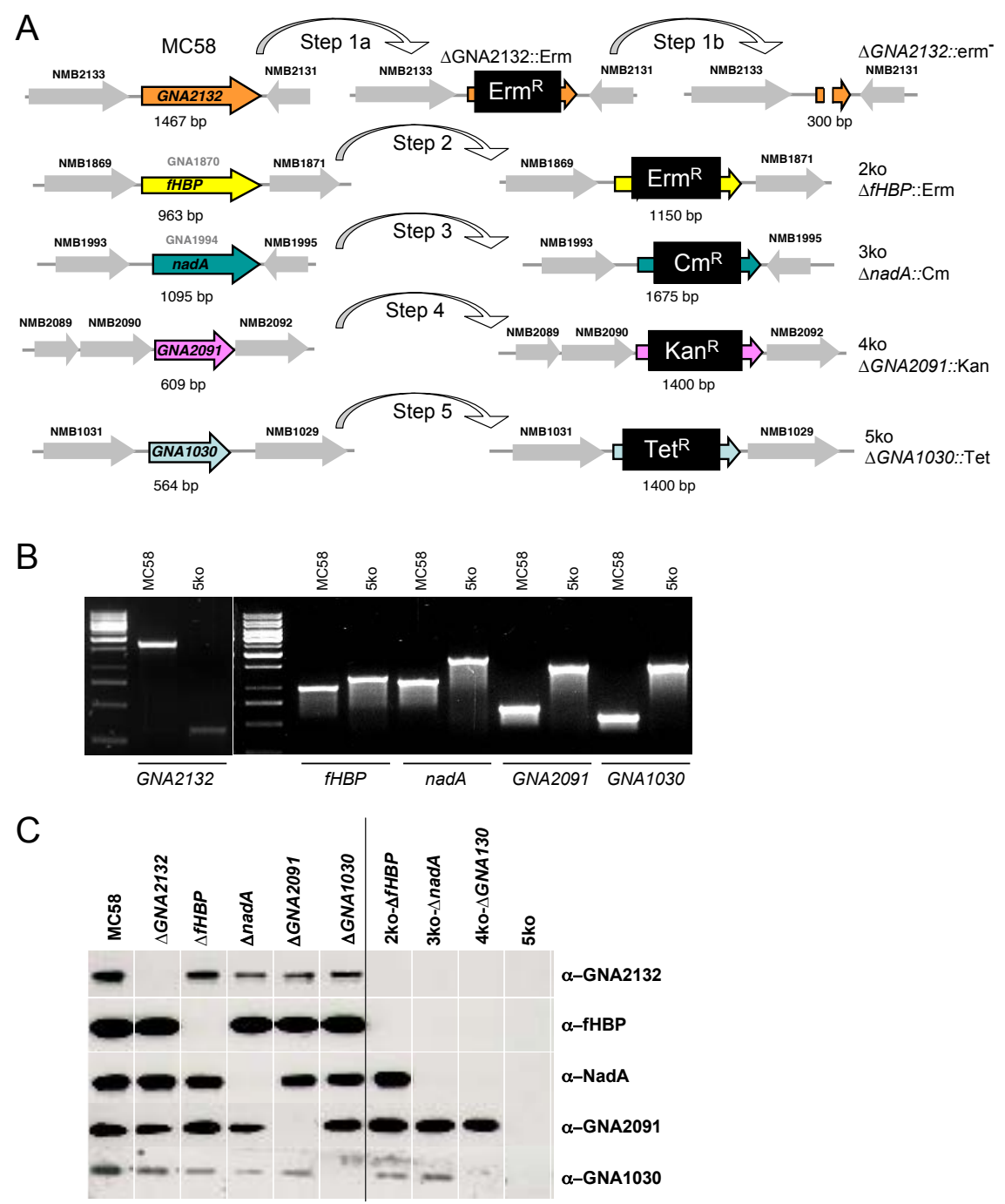

Figure 1. (A) Schematic representation of the stepwise generation of the three (3KO) and five (5KO) protein knockout strains of MC58. (B) PCR verification of the deletion of each gene in the MC58 5KO strain. The expected sizes of the PCR products from the parental MC58 strain and each of the five loci are shown in panel A. (C) Western Blot verification of the absence of each protein in the single, intermediate and final knockout strains. 
A

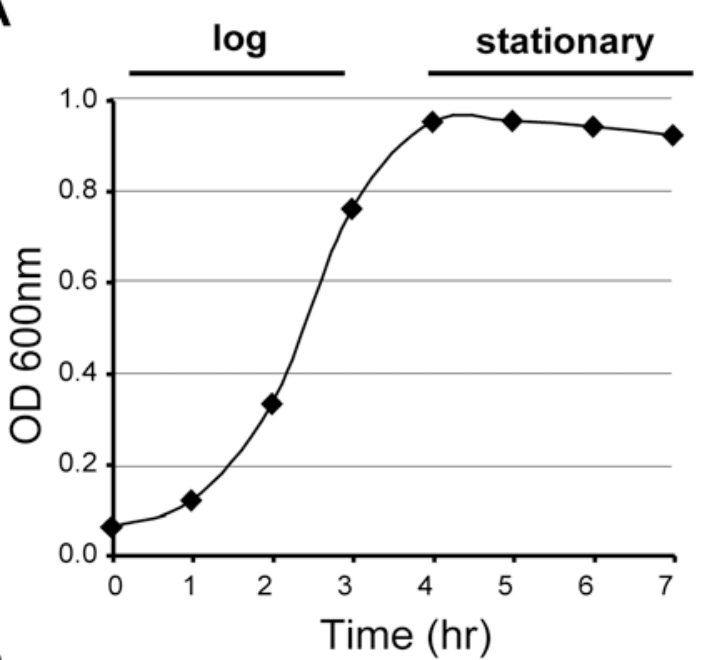

B

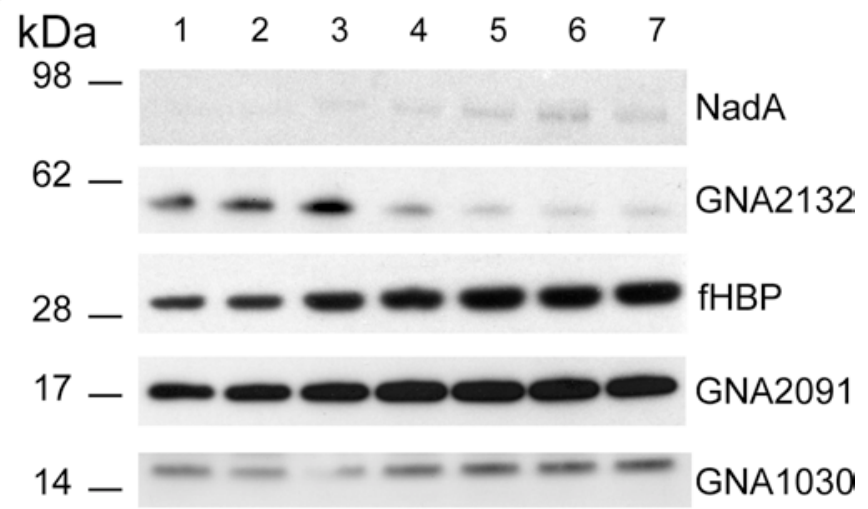

Figure 2. Expression of MenB vaccine antigens in MC58. (A) Time course of growth of the MC58 WT strain. (B) Western blot analysis of total cell extracts of meningococcal cultures, taken at points shown in A along the time course, showing differential growth-phase expression of the MenB vaccine antigens. The approximate molecular weights of the proteins are indicated on the left of each panel. 
A
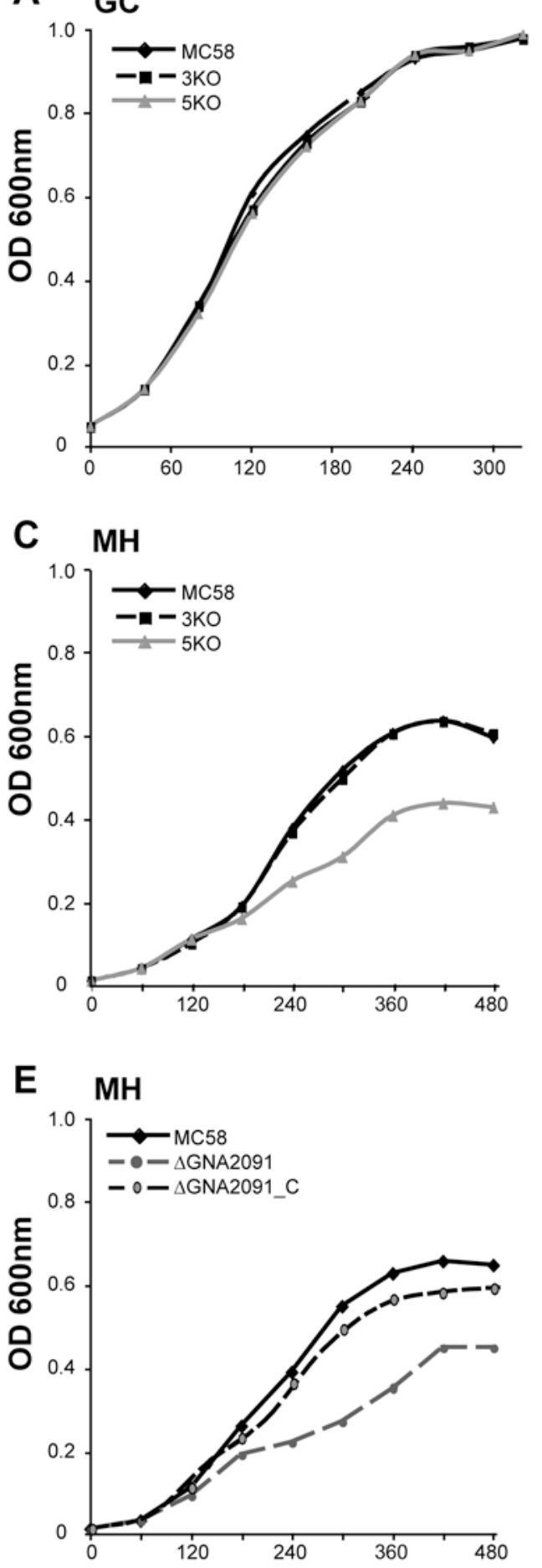

B

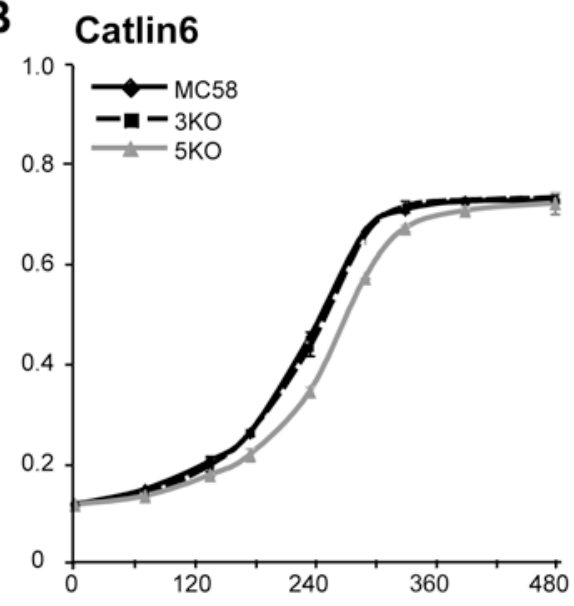

D $\mathrm{MH}$

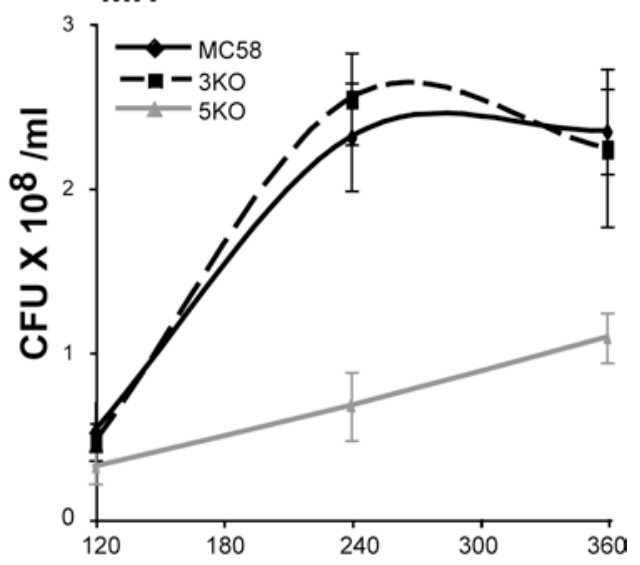

$\mathbf{F}$

$\mathrm{MH}+$ glucose

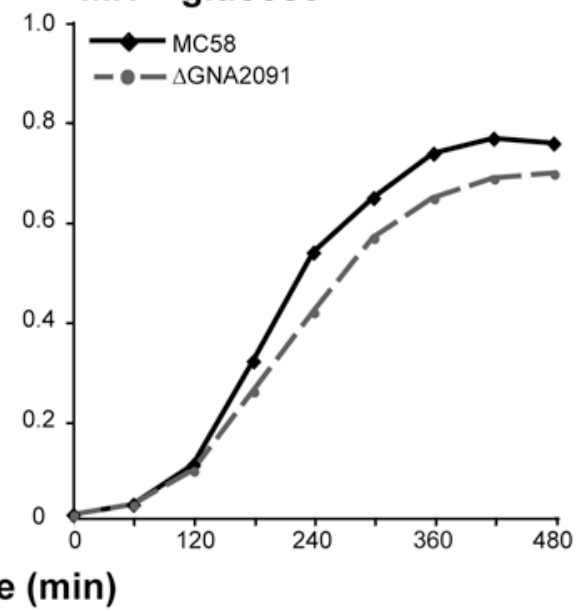

Figure 3. Growth rates of MC58 $\mathrm{WT}, 3 \mathrm{KO}, 5 \mathrm{KO}$ and individual mutant strains in various media. Graphs show growth of MC58, 3KO and 5KO strains grown in (A) GC, (B) Catlin6, and (C-D) Mueller Hinton (MH) broth. MC58 WT, $\Delta$ GNA2091, and the complemented strain $\Delta$ GNA2091_C in (E) $\mathrm{MH}$ broth and (F) $\mathrm{MH}$ broth supplemented with $0.25 \%$ glucose. Growth rates were assessed by optical density (OD) at $600 \mathrm{~nm}$, or colony forming units per $\mathrm{ml}(\mathrm{CFU} / \mathrm{ml})$ in panel $\mathrm{D}$, over time. 
A
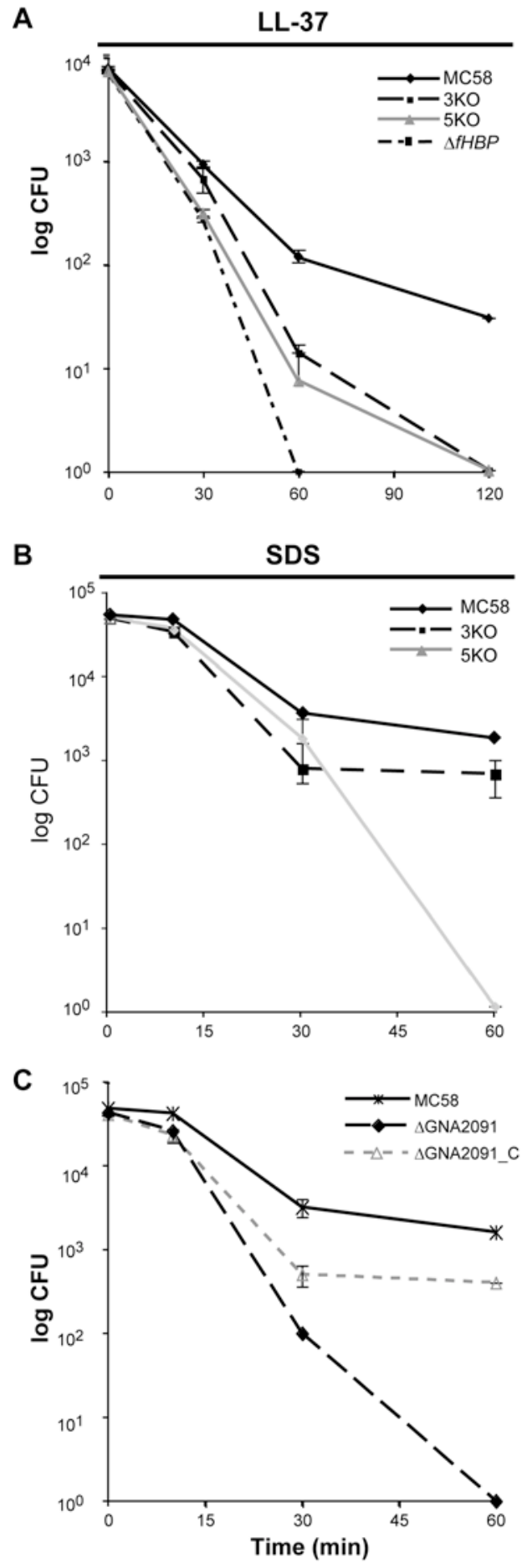

Figure 4. Survival of MC58 WT, 3KO, $5 \mathrm{KO}$ and individual mutant strains during in vitro stress assays. (A) Survival of MC58, 3KO, 5KO and $\Delta \mathrm{fHBP}$ strains in the presence of $3.5 \mu \mathrm{M}$ LL-37. (BC) Survival of MC58, 3KO, 5KO, $\triangle \mathrm{GNA} 2091$, and $\triangle \mathrm{GNA} 2091 \_\mathrm{C}$ strains in the presence of $0.01 \%$ SDS. Experiments were performed in triplicate on several occasions and representative results are shown. Error bars indicate \pm 1 standard deviation of the mean. P values using Student's T-test for the survival of the mutant strain with respect to the isogenic WT parent strain at 60 min are: panel (A) $\leq 0.01$ for all strains; (B) 0.01 for $5 \mathrm{KO}, 0.045$ for $3 \mathrm{KO}$; (C) 0.01 for $\Delta$ GNA2091, 0.023 for $\Delta$ GNA2091_C. 

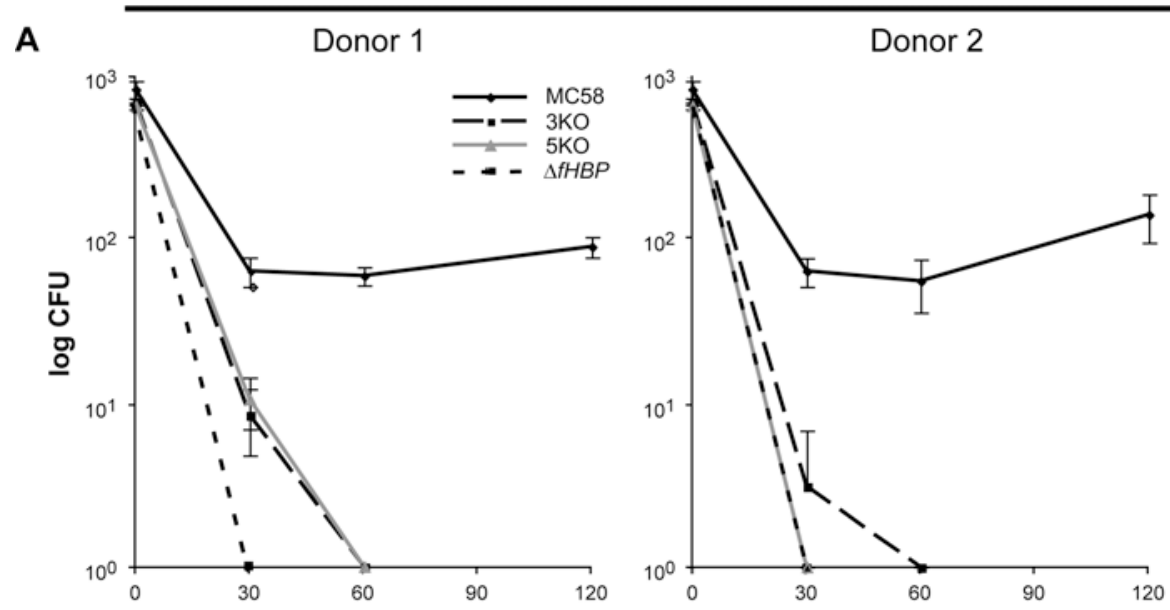

B

Donor 1

Donor 2
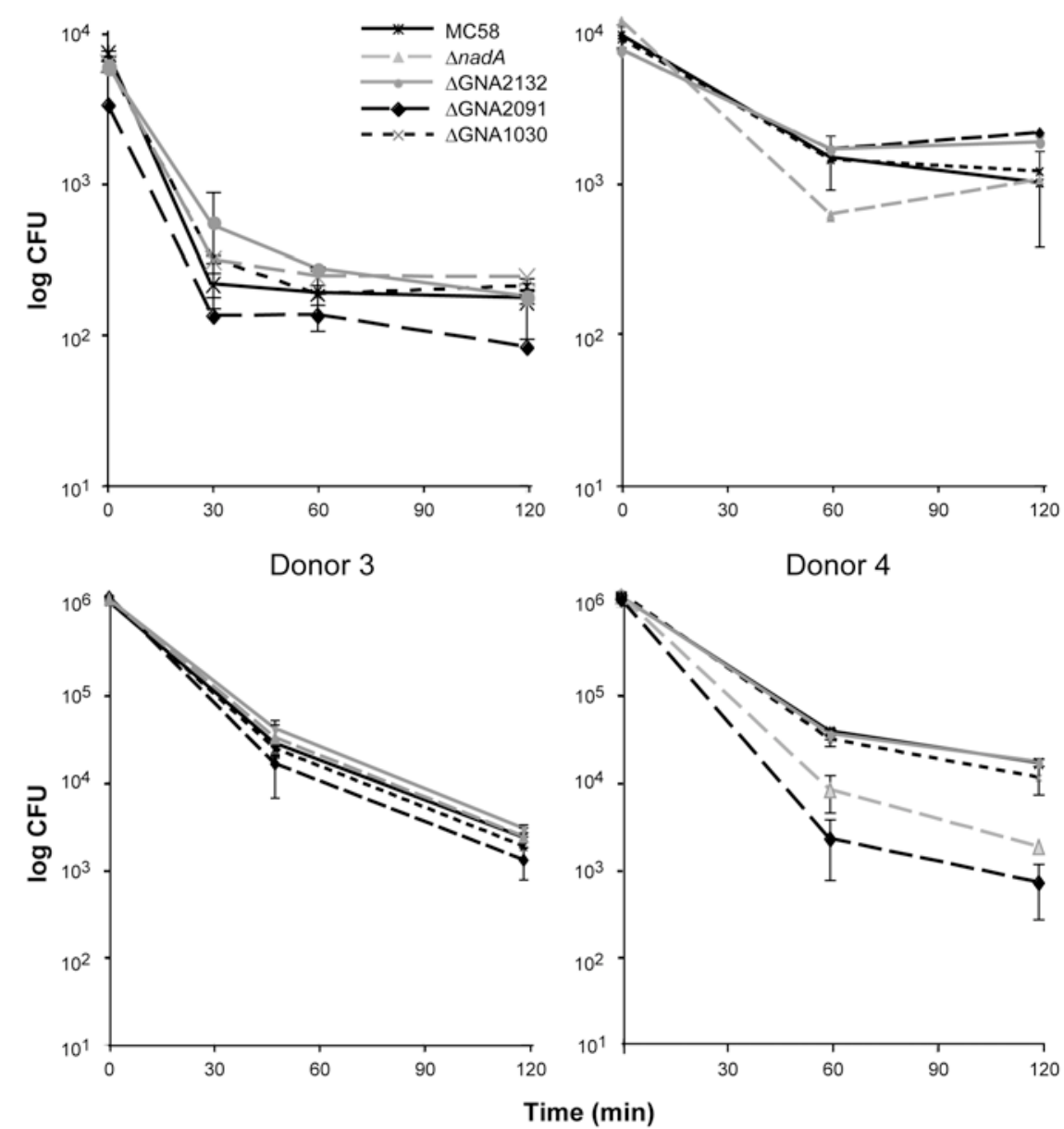

Figure 5. Survival of MC58 WT, 3KO, 5KO, and individual mutant strains in an ex vivo human whole blood model of meningococcal bacteremia. Survival of (A) MC58, 3KO, 5KO and $\Delta \mathrm{fHBP}$ in blood from two different donors; and (B) MC58, $\Delta$ GNA2091, $\Delta$ GNA2132, $\Delta$ nadA, and $\Delta$ GNA1030 in blood from four different donors. Experiments were performed in triplicate on several occasions and representative results are shown. Error bars indicate \pm 1 standard deviation of the mean. $\mathrm{P}$ values using Student's T-test for the survival of the mutant strain with respect to the isogenic WT parent strain at 120 min are: panel $(A) \leq 0.001$ for strains; $(B) \geq 0.05$ for all strains except donor 1 $\Delta$ GNA2091 (0.017) and donor $4-\Delta n a d A$ and $\Delta$ GNA2091 (0.001). 
A

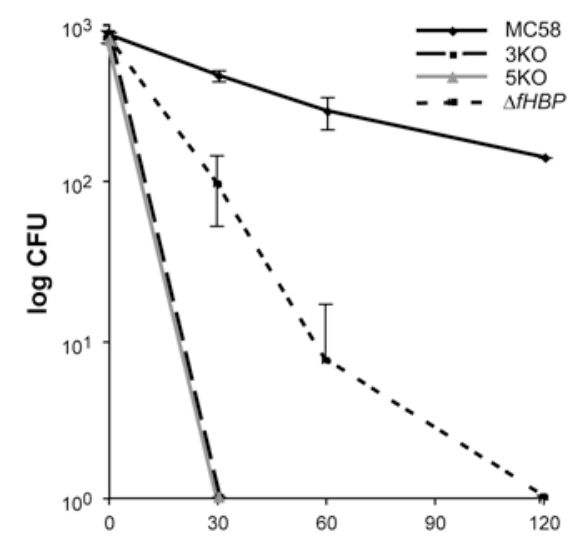

B
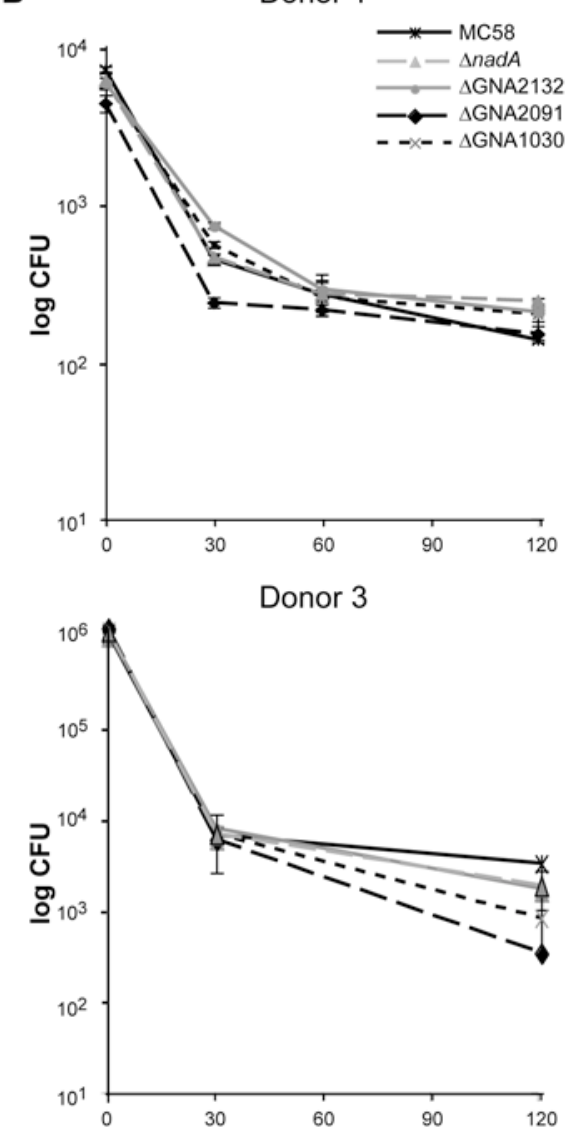

Donor 2

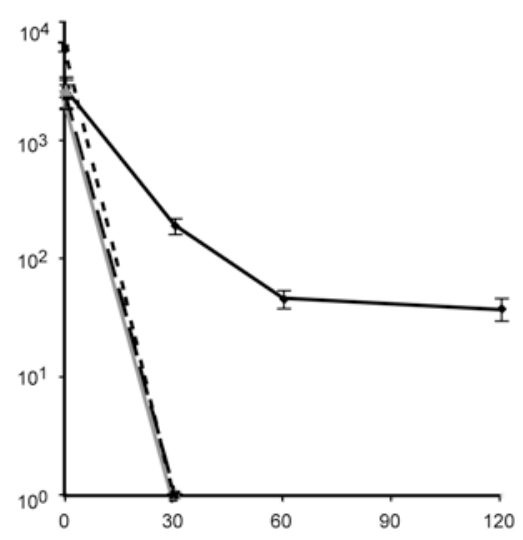

Donor 2
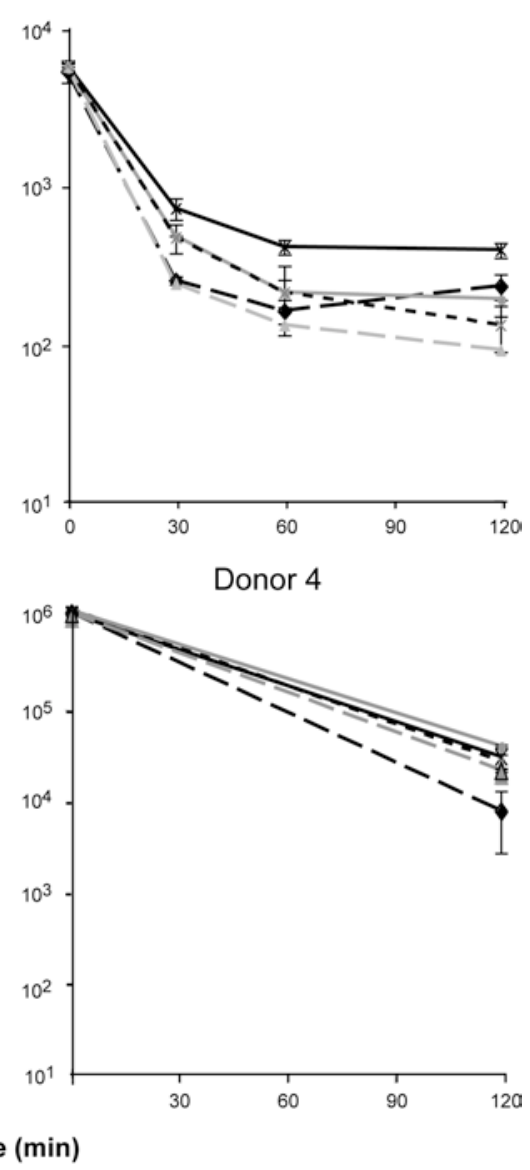

Figure 6. Survival of MC58 WT, $3 \mathrm{KO}, 5 \mathrm{KO}$, and individual mutant strains in an ex vivo human serum model of meningococcal bacteremia. Survival of (A) MC58, 3KO, $5 \mathrm{KO}$ and $\Delta \mathrm{fHBP}$ in serum from two different donors; and (B) MC58, $\Delta$ GNA2091, $\Delta$ GNA2132, $\Delta$ nadA, and $\Delta$ GNA1030 in serum from four different donors. Experiments were performed in triplicate on several occasions and representative results are shown. Error bars indicate \pm 1 standard deviation of the mean. P values using Student's T-test for the survival of the mutant strain with respect to the isogenic WT parent strain at 120 min are: panel (A) $\leq 0.01$ for strains; $(B) \geq 0.06$ for all strains except donor 2 $\Delta$ nadA (0.011), $\Delta$ GNA2132 (0.042), $\Delta$ GNA1030 (0.026), donor 3 all strains $\leq 0.07$, and donor 4 $\Delta$ GNA2091 (0.02). 


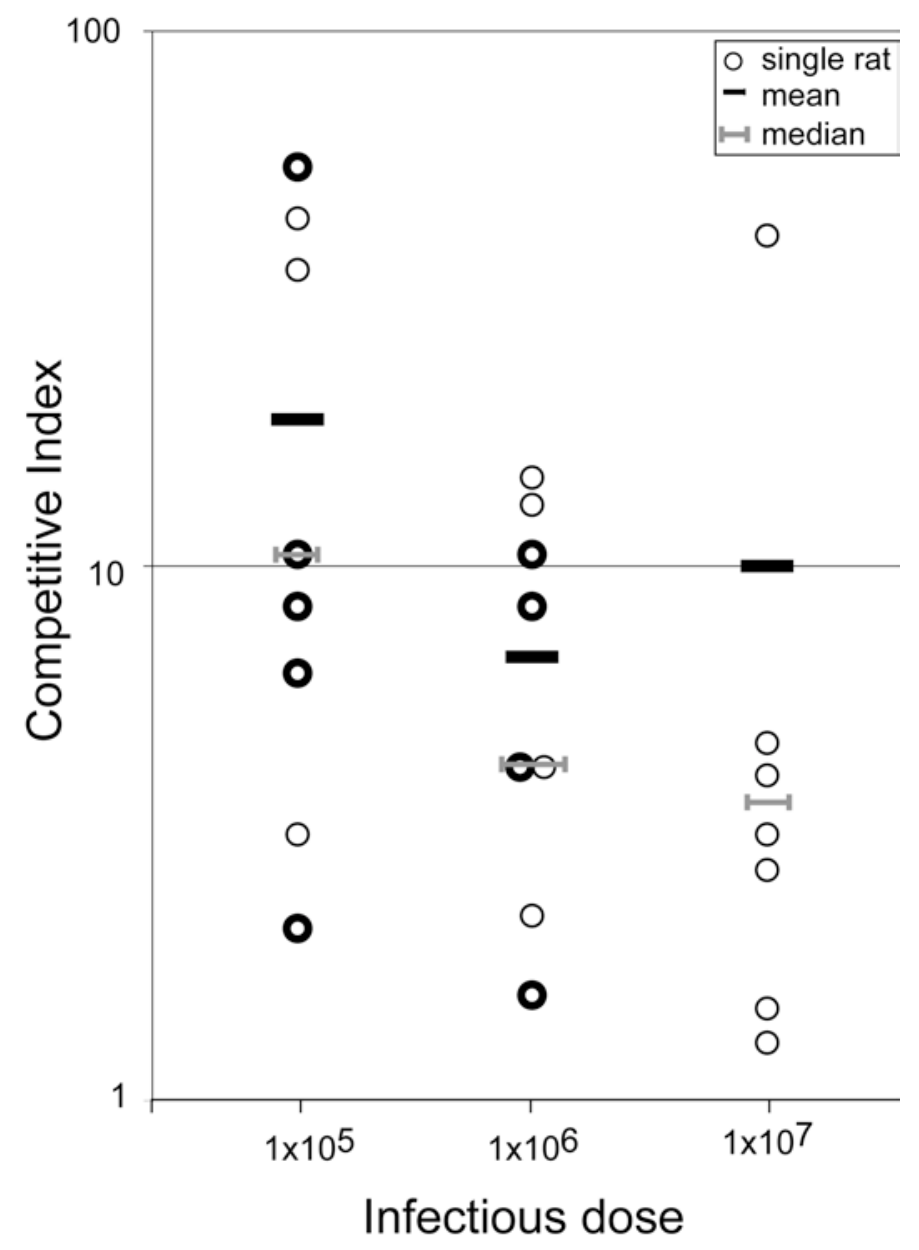

Figure 7. Eight 6 day-old infant rats were infected intraperitoneally with $1 \times 10^{5}, 1 \times 10^{6}$ or $1 \times 10^{7}$ CFU N. meningitidis MC58 WT and 5KO strains at a 1:1 ratio. At $18 \mathrm{~h}$ post-injection blood was collected and serial dilutions were plated and bacterial colonies were counted. Individual CIs are shown for 8 individual animals for the dose of $1 \times 10^{5}$ and $1 \times 10^{6}$, and 7 animals for $1 \times 10^{7}(1$ animal died during the experiment), and the mean and median are plotted for each infectious dose. Bold circles indicate underestimated values ( 0 erythromycin-resistant colonies were isolated, and given a number of 1 in order to calculate the CI). P values using the Wilcoxon Signed-Ranks Test for the survival of the mutant strain with respect to the isogenic WT parent strain are $0.01,0.01$ and 0.02 for the dose of $1 \times 10^{5}, 1 \times 10^{6}$ and $1 \times 10^{7}$, respectively. 